\title{
Dislocation structures in the gamma prime-phase of CMSX-4 and TMS75 after degradation under service conditions
}

\author{
Thomas Link ${ }^{1, a}$, Alexander Epishin ${ }^{1}$, Nils Nawrath ${ }^{1}$, Catrina Michel ${ }^{1}$, \\ Mohamed Nazmy ${ }^{2 b}$ \\ ${ }^{1}$ TU Berlin, I. f. Material Science Technology, Ernst-Reuter-Platz 1, 10587 Berlin, Germany \\ ${ }^{2}$ ALSTOM, Brown Boveri Str. 7, 5401 Baden, Switzerland \\ athomas.link@tu-berlin.de, ${ }^{b}$ mohamed.nazmy@power.alstom.com
}

Key words: Superalloys, SC-material, degradation, dislocation mechanisms, TEM

\begin{abstract}
The single crystal superalloys CMSX-4 and TMS75 were tested for very long times at high temperatures in laboratory creep tests and in service. The microstructure was investigated in TEM and SEM. It was found, that the $\gamma^{\prime}$-phase is cut at stresses much below the threshold given in literature. Cutting takes place by $<110>$ screw dislocations. These mobile dislocations can be locked locally by a Giamei-lock. Thus a stacking fault dipole forms between the locked segment and the preceding ones. The dipole consists of two supershockley dislocations with $30^{\circ}$ character, which are also stabilized by Giamei locks. The dipole formation is analyzed and discussed in detail.
\end{abstract}

\section{Introduction}

Nickelbase superalloys are applied for blades in turbines. Such blades were first used in military engines, then in civil aircrafts and now in stationary turbines. Thus the long-run behavior becomes more and more important. Laboratory investigations however are mostly performed in the several hundreds hour range, which is about a factor of 10 below realistic conditions. It is well known, that the microstructure of superalloys is not stable during high temperature creep: the cubical $\gamma^{\prime}$ precipitates coalesce and grow [1], dislocations relax coherency stresses and generate internal stresses on their part [2]. A central question is, how plastic deformation continues, when dislocation movement, starting in the matrix channels, is blocked by back stresses, built up by preceding dislocations. Cutting of the precipitates becomes necessarily the next step and therefore the topic of the paper is dislocation movement in the $\gamma^{\prime}$-phase of technical alloys under service conditions.

\section{Materials and methods}

Three different sources for the investigated materials were used (Table 1):

(1) Turbine blades of CMSX-4, supplied by Siemens, England. They had completed 12700h operation with 200 starts. The blade was uncooled (massive) and had a Sermaloy 1515 surface coating. The length of the blade from platform to tip was between 26 and $29 \mathrm{~mm}$. Specimens were taken close to the tip, in the middle and close to the platform.

(2) Degraded cylindrical specimens of CMSX-4 and TMS75, supplied by Alstom, Switzerland. The term degradation denominates creep under high temperature and low stress for long times.

(3) Cylindrical creep specimens, tested at the Federal Institute for Materials Research and Testing, BAM, Berlin.

The test parameters are given in Table 1, the material composition in Table 2. Raft formation was quantified on $\gamma$ '-etched surfaces with a LEO GEMINI 1530VP SEM, the dislocations analyzed in a Philips CM30 TEM. For determination of line vectors the TOCA software [3] was used, for Burgers vector identification image simulations were performed by help of the software CUFOUR [4]. 


\section{Results}

The degradation of the $\gamma / \gamma^{\prime}$-morphology in the blade followed the temperature distribution. Close to the platform the cuboidal $\gamma^{\prime}$ morphology was mostly sustained, in the middle rafts had formed perpendicular to the blade axis and in the top (Fig. 1) coarsening was most advanced. On the suction side (convex) of the blade however the rafts are parallel to the blade axis (Fig. 1a), on the pressure side mostly perpendicular (Fig. 1c) and in the center in-between both (Fig. 1b). The stress $(\sigma)$, temperature $(\mathrm{T})$ and time $(\mathrm{t})$ dependence of the raft formation is presented in [1].

Table 1. Tests performed. In blades the local strain $\varepsilon$ is unknown. Temperatures in the blade were measured by pyrometer, stresses calculated.

\begin{tabular}{|c|c|c|c|c|c|c|}
\hline source & material & specimen & $\mathrm{T}\left[{ }^{\circ} \mathrm{C}\right]$ & $\sigma[\mathrm{MPa}]$ & $\mathrm{t}[\mathrm{h}]$ & $\varepsilon[\%]$ \\
\hline \multirow{3}{*}{ Siemens } & \multirow{3}{*}{ CMSX4 } & blade tip & 980 & 30 & 12700 & \\
\hline & & blade middle & 920 & 110 & 12700 & \\
\hline & & blade platform & 800 & 180 & 12700 & \\
\hline \multirow{4}{*}{ Alstom } & \multirow{3}{*}{ TMS 75} & \multirow{4}{*}{ cylindrical } & 1000 & 105 & 7395 & 2.4 \\
\hline & & & 1000 & 90 & 2448 & 0.26 \\
\hline & & & 1050 & 85 & 1272 & 0.3 \\
\hline & CMSX-4 & & 1050 & 38 & 8551 & 0.08 \\
\hline \multirow{3}{*}{$\begin{array}{l}\text { TU-Berlin, } \\
\text { BAM-Berlin }\end{array}$} & \multirow{3}{*}{ CMSX4 } & \multirow{3}{*}{ cylindrical } & \multirow{3}{*}{950} & 180 & 1101 & 0.2 \\
\hline & & & & 180 & 1682 & 0.75 \\
\hline & & & & 180 & 1840 & 1.5 \\
\hline
\end{tabular}

Table 2. Composition of the alloys used, in wt $\%$.

\begin{tabular}{|l|l|l|l|l|l|l|l|l|l|l|}
\hline Alloy & $\mathrm{Ni}$ & $\mathrm{Cr}$ & $\mathrm{Co}$ & $\mathrm{Mo}$ & $\mathrm{W}$ & $\mathrm{Ta}$ & $\mathrm{Re}$ & $\mathrm{Al}$ & $\mathrm{Ti}$ & $\mathrm{Hf}$ \\
\hline CMSX-4 & bal & 6.5 & 9 & 0.6 & 6 & 6.5 & 3 & 5.6 & 1 & 0.1 \\
\hline TMS75 & bal & 3 & 12 & 2 & 6 & 6 & 5 & 6 & - & 0.1 \\
\hline
\end{tabular}
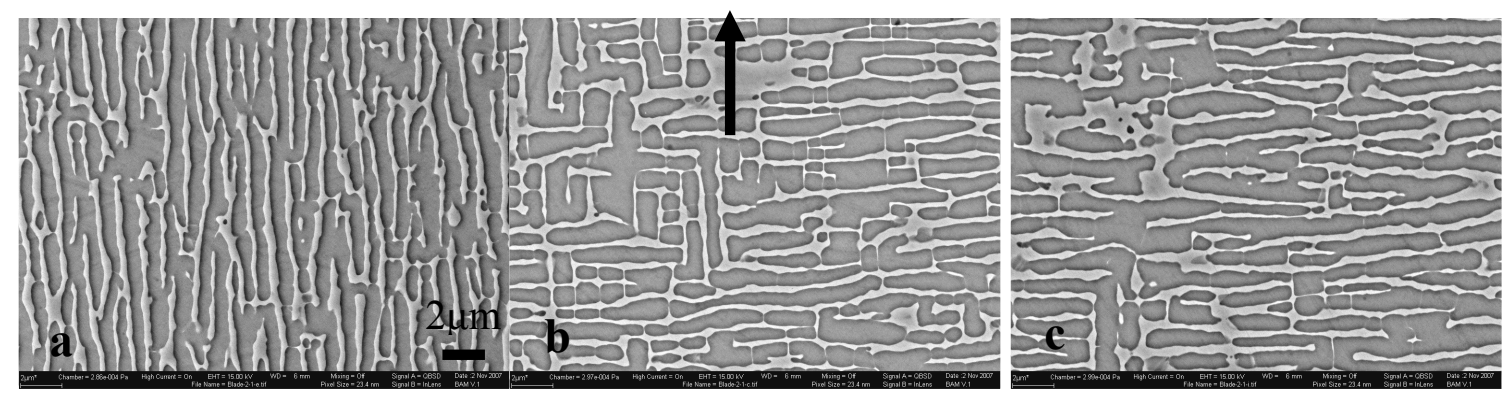

Fig. 1. Longitudinal sections through a CMSX-4 blade, close to the blade tip. (a) suction side, (b) center, (c) pressure side. The direction of the centrifugal force is marked by an arrow.

Representative TEM images of the dislocation structure in the $\gamma^{\prime}$-phase are given in Fig. 2. In the top and middle region the rafts are densely and homogeneously filled with short dislocations (Fig. $2 \mathrm{a}, \mathrm{b})$, having $<110>$ directions. In the bottom region dislocations are restricted to $\{111\}$ shear bands cutting through many $\gamma^{\prime}$ - cubes (Fig. 2c). 

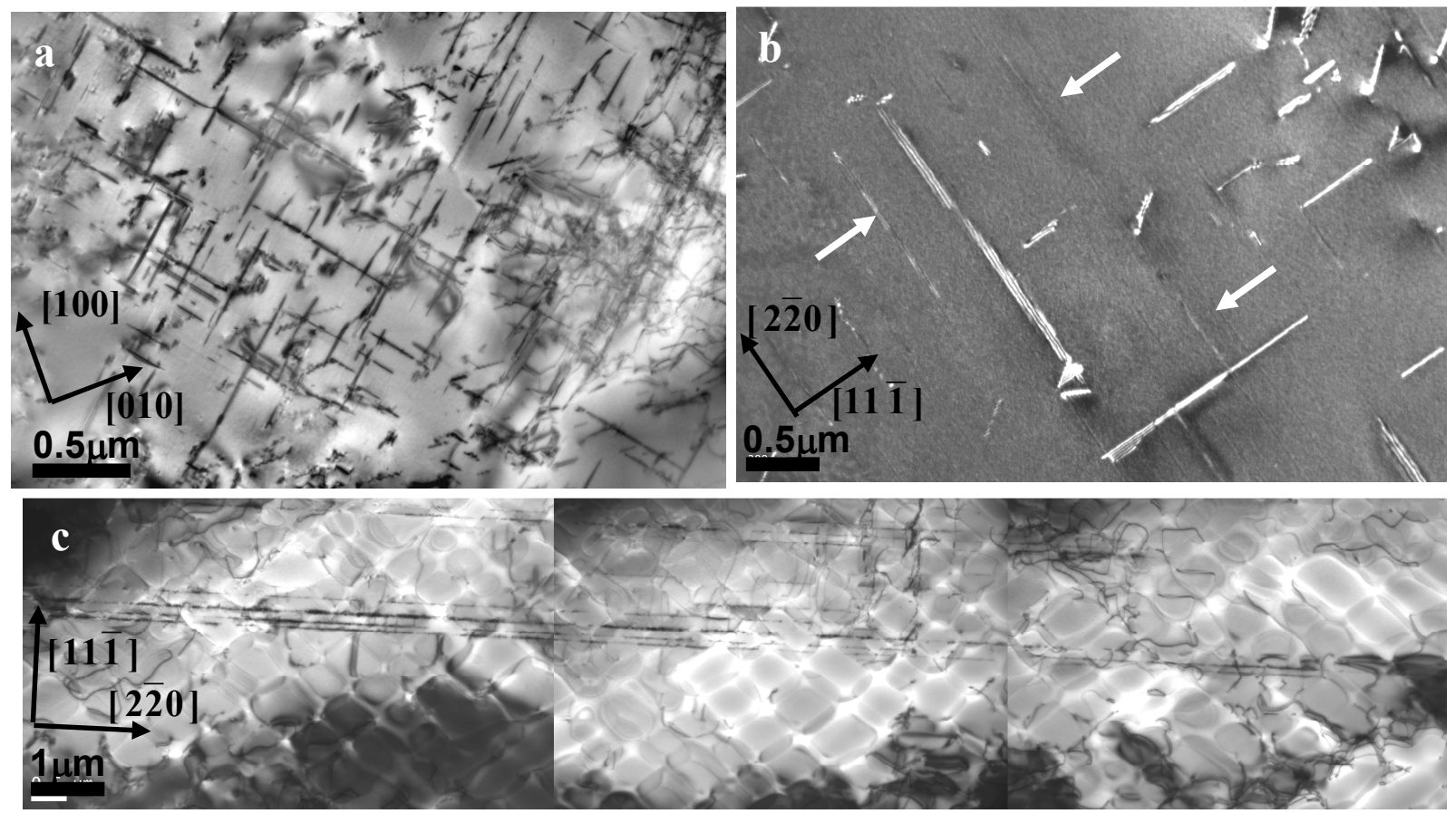

Fig. 2. Dislocations in the $\gamma^{\prime}$-phase of the blade. All images from cross sections. (a) tip, (b) middle, (c) close to platform.

A longitudinal section corresponding to Fig. $2 b$ is presented in Fig. 3a. Double lines parallel to the $\gamma / \gamma$ '-interface with oscillating contrast are visible and a diagonal line. In both cases this gives $<110>$ line vectors. Fig. $3 b$ serves for comparison with a degraded TMS-specimen. Here the matrix channels appear black due to the high dislocation density, which is also high in the rafts. The dislocation geometry inside $\gamma^{\prime}$, parallel and diagonal to the rafts, is similar to that in Fig. 3a, but now also segments perpendicular to the interface are found. In Fig. 3a the area in-between the double lines shows no contrast, in Fig. 3 b it appears as a stacking fault (SF).
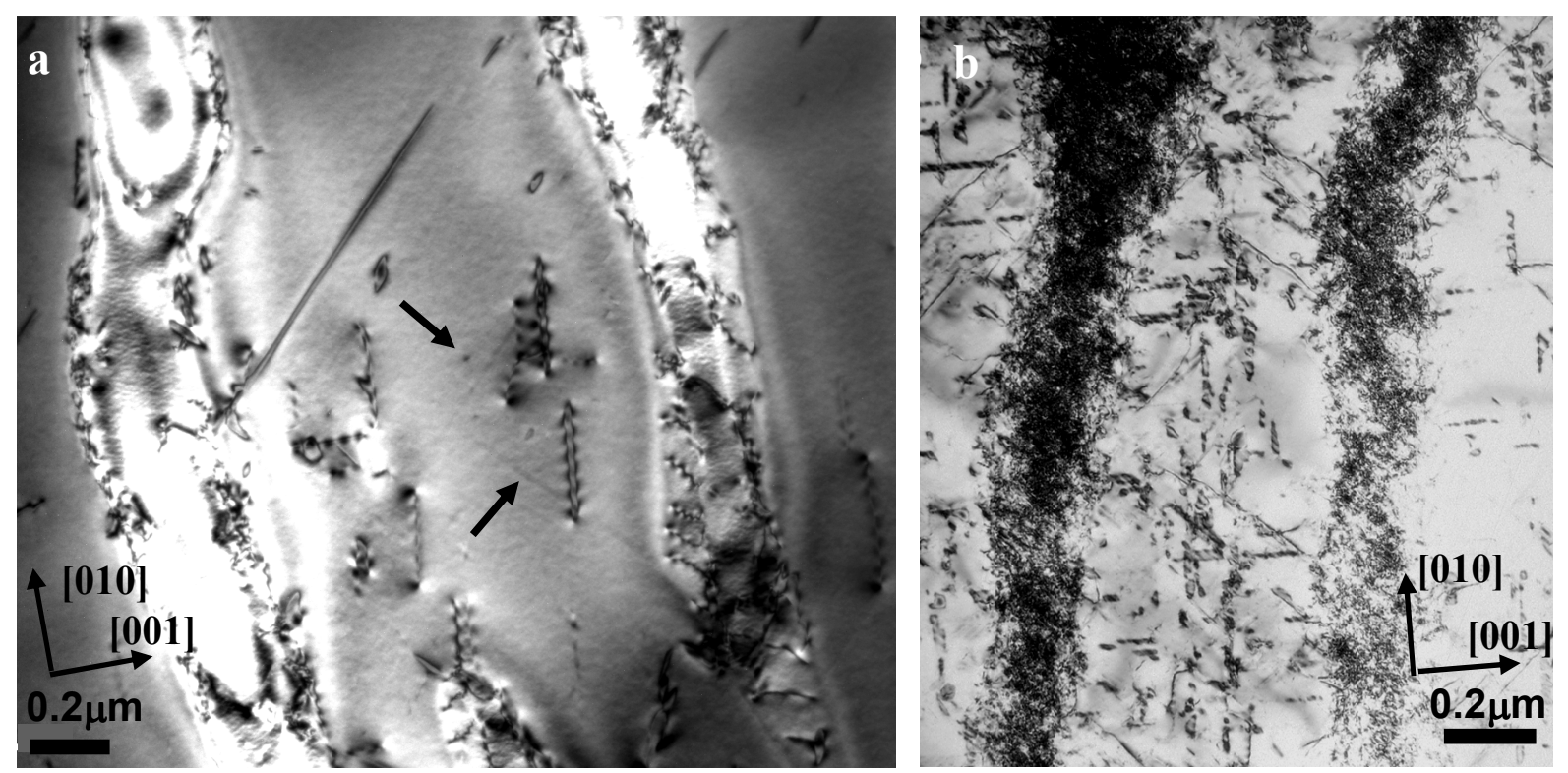

Fig. 3. Longitudinal sections of CMSX-4 (a) and TMS-75 (b). (a): $920^{\circ} \mathrm{C}, 110 \mathrm{MPa}, 12700 \mathrm{~h}$. (b): $1050^{\circ} \mathrm{C}, 85 \mathrm{MPa}, 1272 \mathrm{~h}$ 


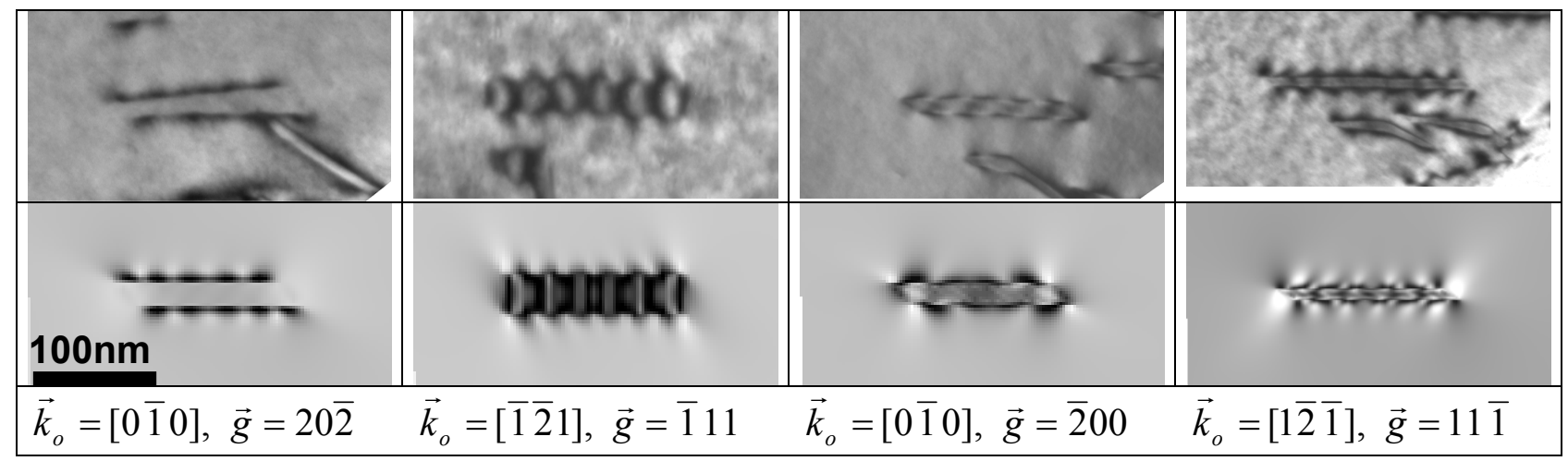

Fig. 4. Dislocation dipoles in CMSX-4, creep deformed $950^{\circ} \mathrm{C}, 180 \mathrm{MPa}, 1840 \mathrm{~h}$.

In each column a TEM micrograph is presented, the simulated image and the imaging conditions with beam direction $\vec{k}_{o}$ and reflection $\vec{g}$. Foil normal is $[0 \overline{1} 0]$.

This effect was analyzed in detail in another specimen (Fig. 4). The double lines were identified as super lattice intrinsic SF dipoles with line vector $\vec{u}=[110]$ and Burgers vector $\vec{b}= \pm 1 / 3[\overline{2} \overline{1} 1]$, giving $30^{\circ}$ dislocations. The formation of these dipoles is illustrated by the following images. Fig. 5a shows, that the observed dipoles are segments of a narrow SF band, which partially collapsed. Fig. 5b shows $<110>$ screw dislocations cutting through the rafts (numbers $1,2,3$ ). The vertical arrows mark the trace of a glide band lying edge on. It extends from the interface in the lower left to the opposite interface in the upper right. Careful inspection of other images like Fig. $2 \mathrm{~b}$ and Fig. 3a reveals, that such faint, straight lines (see arrows) are a general phenomenon in specimens deformed under these conditions. It could be shown that these lines are no APB (anti phase boundary) tubes as described by [5], because the invisibility criterion given there does not apply in our case.
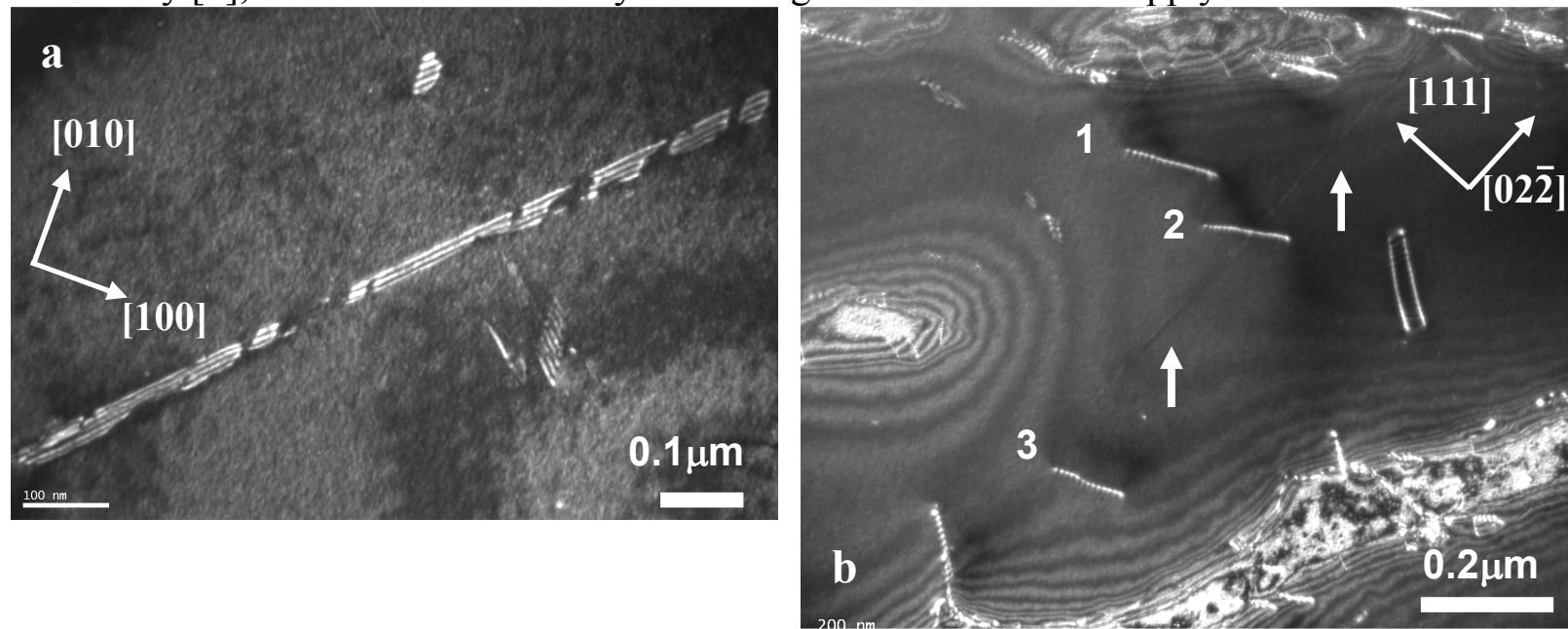

Fig.5 (a) SF band, partially collapsed. Cross section, top of blade. (b) $<110>$ screw dislocations (13 ) and traces of dislocations (arrows). Longitudinal section of CMSX-4, $950^{\circ} \mathrm{C}, 180 \mathrm{MPa}, 1101 \mathrm{~h}$.

Inspection of all TU/BAM specimens (Table 1) gave, that SF bands always appear together with such $<110>$ screw dislocations.

In order to check, whether a correlation exists between $\gamma^{\prime}$-morphology change and dislocation activity in the $\gamma^{\prime}$-phase, the $\gamma^{\prime}$-morphology and $\gamma^{\prime}$-dislocation density were characterized in TU/BAM specimens. This was performed in a rather pragmatic way, facing the large number of micrographs and the variation of the structures within one specimen. Five states for the morphology were introduced: cuboidal (1), fully rafted (2), topologically inverted (3), transition between (1) and (2) and transition between (2) and (3). The dislocation densities were divided into: no dislocations (0), few (1) middle (2) and many (3). These conditions were characterized by representative images and the other images evaluated relative to these standards. The complete evaluation was performed by the same person. The result is shown in Fig. 6a. 

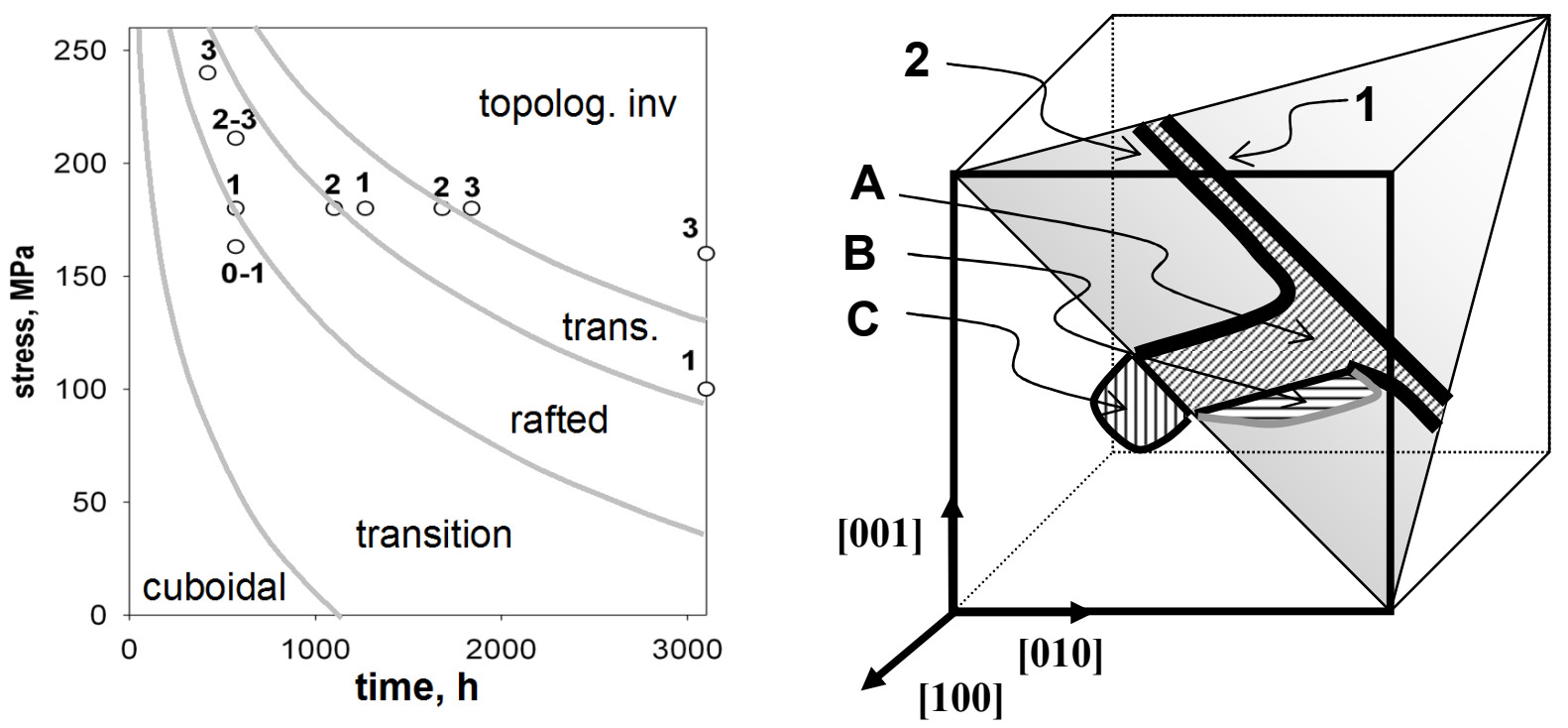

Fig. 6. (a) Change of the $\gamma / \gamma^{\prime}$-morphology in CMSX-4 at $950^{\circ} \mathrm{C}$ as function of stress and time.

The $\gamma^{\prime}$ dislocation density is given as a number at the regarding point, running from 0 (no dislocations) up to 3 (many). (b) Giamei locks on a dipole. The screw super dislocation [011] is

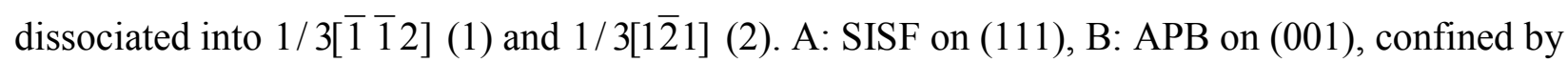

$1 / 2[1 \overline{1} 0]$ (straight) and $1 / 6[\overline{1} \overline{1} 2]$ (curved), C: APB on (100), confined by $\mathbf{1} / 2[\mathbf{0} \overline{1} 1]$ and $1 / 6[2 \overline{1} \overline{1}]$. Stress direction is [001].

\section{Discussion}

Such SF loops were first found in $\mathrm{Ni}_{3} \mathrm{Al}$ by Staton-Bevan in 1975 [6]. Since then many authors reported this phenomenon and discussed it mostly under two aspects: (1) Is the dislocation pair a super dislocation dissociated in super shockleys [7, 8] or a dipole [7-10]? (2) Was the pair generated by the reaction between two dislocations $[8,11]$ or by a local lock in a single dislocation $[9,10]$ ? In our case we find good agreement with Y.Q. Sun e. a [9], who explain the formation and shape of the loop by the Giamei locking mechanism [12] in a single dislocation. In this case a moving $<110>$ super dislocation does not dissociate locally into two $1 / 2<110>$ partials as in the Kear Wilsdorf lock [13] but into two super shockleys (Fig. 6b). The trailing super shockley splits once more into a Shockley edge dislocation and a $1 / 2<110>$ screw dislocation, which can cross slip from the $\{111\}$ glide plane into the $\{100\}$ cross slip plane, driven by the anisotropy of the APB energy. Thus the glissile dislocation is locally transformed into a nonplanar, i.e. locked configuration. The trailing dislocation however is simultaneously locked to the leading one by the energy of the super lattice intrinsic SF (SISF). The result is the bowing out of the trailing dislocation. As soon as a segment of this bow becomes parallel to $<110>$ it can form another Giamei lock in analogous manner along this direction. Typical for such a Giamei locked super shockley partial is the $30^{\circ}$ angle between line and Burgers vector, as found in our case.

Because the Burgers vectors of the partials, confining a Giamei lock, are perpendicular, there is no repulsion between these dislocations, contrary to the two parallel Burgers vectors of a KearWilsdorf lock. Therefore Giamei locks are less stable, i.e. they play only a role under low applied stresses. The fact, that the SF band is partially collapsed in Fig. 5a illustrates this low stability.

When the stress has the direction [001] exclusively super dislocations with Burgers vectors with non zero z-component experience a shear stress. The consequence is, that the dipoles are extended in $<110>$ directions perpendicular to the stress direction, for example [ $\overline{1} 10]$ in Fig. 6b. It follows, that in TEM observations of cross-sections the images of the dipoles must have $<110>$ directions parallel to the foil surface as in Fig. 2a and 5a (foil untilted), on longitudinal sections parallel to the 
$\gamma / \gamma^{\prime}$-interface (Fig. 3a). But this rule does not apply in each case: especially in the highly deformed specimens from Alstom and Siemens (Table 1) one finds counter-examples. This is reasonable however, because the local stress fields, acting on a certain dislocation are not simply uniaxial, especially when temperature gradients are generating thermal stresses comparable with the applied stress. That this can be the case follows from Fig. 1a, where the $\gamma / \gamma^{\prime}$ interface is no more perpendicular to the blade axis.

Another indication for internal stresses follows from Fig. 6a. Besides the expected result, that $\gamma^{\prime}-$ cutting only starts, when rafting is completed, it was found, that the external load, necessary for $\gamma^{\prime}-$ cutting, decreases with time and is much lower than about $450 \mathrm{MPa}$, as supposed according to literature [14]. The threshold for $\gamma^{\prime}$-cutting arises from the SISF or APB generated, when a matrix dislocation enters the $\gamma^{\prime}$-phase. Because the phase composition does not change during $950^{\circ} \mathrm{C}$ creep, the fault energies are constant and consequently the threshold too. Therefore the reason, that cutting increases with time, must be the matrix dislocations, building up internal stresses, overcoming the interfacial barrier. The raft morphology supports the stress accumulation, because it prevents dislocations from bypassing the $\gamma^{\prime}$-phase.

\section{Conclusion}

Under service conditions the $\gamma^{\prime}$-phase is strongly deformed. Cutting of $\gamma^{\prime}$ occurs by $<110>$ dislocations. At $950^{\circ} \mathrm{C}$ and low stresses in these mobile dislocations locally stable Giamei locks are formed. They cause SF-dipoles, which are left behind as debris, which accumulates and acts as obstacle for $\gamma^{\prime}$-cutting. For long creep times the threshold for $\gamma^{\prime}$-cutting is surmounted for much lower applied stresses than in short term tests, due to internal stresses induced by interfacial dislocations.

\section{References}

1. A. Epishin, T. Link, H. Klingelhöffer, B. Fedelich, U. Brückner and P. D. Portella: Mat. Sci. and Eng. A Vol. 510-511 (2009), p. 262

2. T. Link, A. Epishin, U. Brückner and P. Portella: Acta mater. Vol. 48 (2000), p. 1981

3. S. Zaefferer: Advances in imaging and electron physics Vol. 125 (2002), p. 355

4. R. Schäublin and P. Stadelmann: Mater. Sci. Eng. Vol. A164 (1993), p. 373

5. P.M. Hazzledine and Y.Q. Sun: Mater. Sci. Eng. Vol. A152 (1992), p. 189

6. A.E. Stanton-Bevan and, R.D. Rawlings: Phil. Mag. Vol. 32,4 (1975), p. 787

7. J. Sun, C.S. Lee and J.K.L. Lai: Phys. Stat. Sol. (a) Vol. 158 (1996), p. 369

8. P. Shang, I.P. Jones and R.E. Smallman: Phys. Stat. Sol. (a) Vol. 174 (1999), p. 343

9. Y.Q. Sun, M.A. Crimp and P.M. Hazzledine: Phil. Mag. A Vol. 64,1 (1991), p. 223

10. I. Baker and E.M. Schulson: Phys. Stat. Sol. (a) Vol. 89 (1985), p. 163

11. B.H. Kear, and I.M. Oblak: Journal de Physique, Colloque C7, No 12, Tome 35, (1974), p. 35

12. A.F. Giamei, J.M. Oblak, B.H. Kear and W.H. Rand, Proceedings of the 19th Annual Meeting EMSA, Baton Range: Claitor's Pub. Div. (1971), p. 112

13. B.H. Kear: Acta. metall. Vol. 12 (1964), p. 555

14. T. Tinga, Phd. work, Technische Universiteit Eindhoven, Eindhoven (2009) 